\title{
Dynamic Altitude Control for Motion Coordination in an Estimated Shear Flow
}

\author{
Levi DeVries* and Derek A. Paley ${ }^{\dagger}$ \\ University of Maryland, College Park, MD, 20742, USA
}

\begin{abstract}
Windfields present a challenge to multi-vehicle coordination in applications such as environmental sampling. The presence of a windfield can disrupt inter-vehicle spacing such that the group covers less area, expends energy at a faster rate, strays from a desired formation, or provides irregular measurement data. However, an autonomous or remotely piloted vehicle can also take advantage of the vertical variation of a windfield to maintain a desired inertial speed and to coordinate its motion with other vehicles. This paper presents results for motion coordination in an estimated flowfield with wind shear using a three-dimensional self-propelled particle model with a dynamic altitude control. The paper derives conditions specifying the feasibility of speed-regulated trajectories and Lyapunov-based decentralized control algorithms to stabilize parallel formations in a known, uniform shear flow and symmetric circular formations in a known vortex flow. These algorithms are extended to unknown flowfields by presenting a distributed, recursive Bayesian filter that provides local estimates of the flow from noisy flowspeed measurements, allowing particles to utilize flowfield estimates in the control. Theoretical results are illustrated using numerical simulations.
\end{abstract}

\section{Nomenclature}

$N \quad$ Number of particles in the system

$k \quad$ Particle index; $1, \ldots, N$

${ }^{*}$ Graduate Student, Department of Aerospace Engineering, University of Maryland, and AIAA Student Member.

${ }^{\dagger}$ Associate Professor, Department of Aerospace Engineering and the Institute for Systems Research, University of Maryland, and AIAA Associate Fellow. 
$\vec{r}_{k / O}$ Vector of three-dimensional position of particle $k$ with respect to origin $O$

$r_{k} \quad$ Complex coordinates of $k^{\text {th }}$ particle's horizontal position

$z_{k} \quad$ Altitude of $k^{\text {th }}$ particle

$\dot{r}_{k} \quad$ Complex coordinates of $k^{\text {th }}$ particle's horizontal velocity

$f_{k} \quad$ Complex coordinates of horizontal flow velocity at $r_{k}$

$\theta_{k} \quad$ Orientation of $k^{\text {th }}$ flow-relative horizontal velocity

$\gamma_{k} \quad$ Orientation of $k^{\text {th }}$ total horizontal velocity

$u_{k} \quad$ Flow-relative horizontal steering control of $k^{\text {th }}$ particle

$\nu_{k} \quad$ Total horizontal steering control of $k^{\text {th }}$ particle

$h_{k} \quad$ Flow-relative horizontal speed of $k^{\text {th }}$ particle

$s_{k} \quad$ Total horizontal speed of $k^{\text {th }}$ particle

$\xi_{k} \quad$ Total horizontal speed control of $k^{\text {th }}$ particle

$w_{k} \quad$ Climb rate of $k^{\text {th }}$ particle

$c_{k} \quad$ Instantaneous center of the $k^{\text {th }}$ particle's horizontal trajectory

$W_{\text {ref }}$ Reference wind speed

$z_{r e f}$ Reference altitude

$h_{0} \quad$ Shaping parameter in shear flow model

$\Omega \quad$ Set of flowfield parameters

$\tilde{\alpha} \quad$ Noisy flowfield measurement

A Vector of flowfield measurements

$\sigma_{\alpha}^{2} \quad$ Variance of flowfield measurement noise

$\gamma_{0} \quad$ Reference angle

$s_{0} \quad$ Reference speed

$K_{\gamma} \quad$ Steering control gain

$K_{s p} \quad$ Speed control gain

$i \quad$ Imaginary unit

\section{Introduction}

Unmanned and remotely piloted aerial vehicles are now commonly used as persistent surveillance and environmental sampling platforms. ${ }^{1,2}$ Multiple long-endurance vehicles deployed in a coordinated manner can collect data over vast spatial and temporal domains while simultaneously regulating the sampling densities of their measurements. The duration of a sampling mission is limited by the amount of energy (fuel, batteries, etc.) a vehicle can carry. In addition, external flowfields due to wind impede vehicle motion and disrupt multi-vehicle coordination, causing vehicles to possibly cover less area, expend more energy, 
or provide irregular measurement data.

However, external flows need not always be a disruptive phenomenon. For example, nature provides instances of animals taking advantage of external flowfields in order to travel with greater efficiency: vultures fly in thermals to gain altitude and remain aloft with little to no wing flapping, ${ }^{3}$ and albatross utilize wind shear to travel vast distances without landing. ${ }^{4}$ This paper addresses how multi-vehicle systems may similarly utilize vertical wind shear as a means of maintaining multi-vehicle coordination via altitude control.

The wind shear allowing albatross to soar great distances while expending minimal energy occurs when bodies of air move relative to the ground and one another. ${ }^{5}$ This relative motion creates significant changes in windspeed with altitude. During the process known as dynamic soaring, ${ }^{4}$ albatross utilize stronger windspeeds at higher altitudes to compensate for kinetic energy losses due to aerodynamic drag. In this manner, the bird takes advantage of external wind to control its forward speed such that it can maintain flight. ${ }^{4}$ While albatross use wind shear as a means of maintaining flight over long distances, wind shear can also be used as a means of regulating horizontal speed in order to coordinate the motion of multiple aerial vehicles using only altitude and steering control.

With only speed and turn rate control, decentralized multi-vehicle control algorithms can be used to steer vehicles to sampling formations with desired inter-vehicle spacing in a spatially-varying flowfield. ${ }^{6,7}$ However, the vehicle speed relative to the flow is often assumed to be fixed to maximize endurance. This paper shows that the total horizontal speed can be controlled by utilizing knowledge of the flowfield's vertical flowspeed profile. By varying a sampling vehicle's altitude to achieve a desired total speed over ground, inter-vehicle spacing can be regulated without sacrificing vehicle endurance.

This paper extends a two-dimensional model of self-propelled particle motion to three dimensions by assuming each particle maintains control of its climb rate. In recent years much work has been produced regarding the coordination of multi-vehicle systems in a horizontal plane. In a flow-free setting, Sepulchre et al. ${ }^{8}$ stabilized parallel and circular formations, as well as symmetric circular formations in which particle separation is regulated along the circular formation. In moderate flows, where particle speeds are greater than the magnitude of the flowfield such that forward progress can always be maintained, Paley and Peterson ${ }^{6}$ extended the parallel and circular formation results to motion in a time-invariant flowfield as well as symmetric circular formations in a uniform flowfield. Techy et al. ${ }^{9}$ extended the results to motion around convex loops. Similar work includes that of Frew et al. ${ }^{10}$ who used Lyapunov analysis to generate guidance vector fields in a known flowfield, and Zhang, ${ }^{11}$ who used orbit functions to coordinate motion on smooth curves. Extensions to time-varying flows were made by Peterson and Paley ${ }^{12}$ and to strong flowfields, in which the flowfield magnitude may be greater than the particle speed, were made by DeVries and Paley ${ }^{13}$ as 
well as Bakolas and Tsiotras. ${ }^{14}$

This paper presents a three-dimensional motion model in which each vehicle maintains constant flow-relative speed with respect to the horizontal plane and controls its vertical speed. Justh and Krishnaprasad ${ }^{15}$ utilized a three-dimensional self-propelled particle model and derived multi-vehicle steering control algorithms to stabilize rectilinear, circular, and helical formations. Using a similar model, Scardovi et al. ${ }^{16}$ provided control algorithms stabilizing the same formations under varying communication topologies. Hernandez and Paley $^{17}$ extended the results to three-dimensional motion within a time-invariant flowfield.

In order to regulate vehicle speed in an unknown flowfield, each vehicle must estimate the local wind shear. Prior works have generated flowfield estimates from noisy measurements using nonlinear observer techniques, ${ }^{12}$ a distributed consensus filter, ${ }^{18}$ and a particle filter. ${ }^{19}$ This paper utilizes a distributed, recursive Bayesian filter ${ }^{20}$ to estimate the parameters of an idealized shear flow model. The filter utilizes a nonlinear likelihood function to assimilate noisy measurements of the flowfield and to determine the most probable point in parameter space corresponding to the true flowfield parameters.

The estimation of wind fields has been given much attention in recent years. Many techniques have been utilized to estimate the wind fields affecting aircraft from micro- to macro-scales. At the micro-scale, Palanthandalam-Madapusi et al. ${ }^{21}$ implemented an unscented Kalman filter to estimate wind disturbances. Chao and $\mathrm{Chen}^{22}$ employed multiple UAV's to estimate parameters associated with a partial differential equation approximating a wind field. At the macro-scale, Mulgund and Stengel ${ }^{23}$ implemented an extended Kalman filter to provide wind shear estimates for use in feedback control of aircraft. Lawrance and Sukkarieh ${ }^{24}$ used Gaussian process regression to estimate a wind field for exploration and exploitation of gliding UAV's. In related work, Lynch et al. ${ }^{25}$ utilized decentralized proportional-integral (PI) average consensus estimators coupled with Kalman filtering to model environments using sensor platforms with time-varying communication topologies.

The contributions of this paper are: (1) a model of particle motion in three dimensions with constant (flow-relative) speed in the horizontal plane and corresponding feasibility conditions for desired trajectories of the motion model; (2) decentralized control algorithms that steer particles to feasible equal-speed parallel formations in a time-invariant uniform flowfield with vertical shear and to feasible equal-speed circular formations in which particles are equally spaced in a spatially varying flowfield; (3) a recursive Bayesian filter that estimates the shear flow from noisy flowspeed measurements and an implementation of the decentralized control laws using the estimated shear flow.

The outline of this paper is as follows. Section II presents a model of three-dimensional self-propelled particle motion subject to a time-invariant flowfield with a known vertical profile, assuming the flow magnitude is less than the vehicle speed relative to the flow. It 
also presents the derivation of a coordinate transformation that enables the speed to be indirectly regulated by controlling altitude and feasibility conditions for altitude-induced speed-regulated trajectories. Section III presents a recursive Bayesian filter framework for estimating flowfield parameters using noisy flowspeed measurements. Section IV uses nonlinear control techniques to design decentralized multi-vehicle control algorithms that steer vehicles to a desired formation with a desired speed and provides simulations of each control algorithm in a flowfield with unknown wind shear. Section V summarizes the paper and provides insight into ongoing work.

\section{Particle Dynamics in a Shear Flow}

This section derives a model of three-dimensional vehicle motion in a flowfield where each vehicle travels at constant (flow-relative) speed in the horizontal plane and maintains control of its climb rate. The vehicle model is then used to derive feasibility conditions for speed regulated trajectories.

\section{A. Three-dimensional Self-propelled Particle Model in a Flowfield}

The three-dimensional, self-propelled particle model that is derived in this section extends previous models of self-propelled particle motion in a plane ${ }^{6,8,9,26,27,28}$ by assuming each particle has control of its turn rate and climb rate. Previous works on particle motion in three dimensions by Justh and Krishnaprasad, ${ }^{15}$ Scardovi et al. ${ }^{16}$ and Hernandez and Paley $^{17}$ assume each particle moves at constant (flow-relative) speed in three-dimensions. This paper assumes each particle travels at fixed (flow-relative) speed in the horizontal plane and controls its altitude so as to reach a desired total horizontal speed. Development of the particle model in this section enables the derivation of control algorithms in Section IV.

Consider a collection of $N$ Newtonian particles each able to control its turn and climb rates. The $k^{\text {th }}$ particle's position with respect to a ground-fixed inertial reference frame is represented by the vector $\vec{r}_{k / O}$. When convenient we represent the position of the $k^{\text {th }}$ particle in the horizontal plane using complex coordinates $r_{k}=x_{k}+i y_{k} \in \mathbb{C}$ and the altitude $z_{k} \in \mathbb{R}^{+}$, as shown in Figure 1(a). In the absence of flow, each particle's horizontal motion is controlled by a gyroscopic steering force normal to its horizontal velocity $\dot{r}_{k}$, which has constant magnitude $h_{k}$. The orientation of the $k^{\text {th }}$ particle's horizontal velocity is a point $\theta_{k}$ on the unit circle such that in complex coordinates, the horizontal velocity is $h_{k} e^{i \theta_{k}}=$ $h_{k}\left(\cos \theta_{k}+i \sin \theta_{k}\right)$. A decentralized steering control $^{\mathrm{a}} \dot{\theta}_{k}=u_{k}(\mathbf{r}, \boldsymbol{\theta}, \mathbf{z})$ is designed using state-

\footnotetext{
${ }^{a}$ The subscript is dropped and bold fonts are used to represent an $N \times 1$ matrix, e.g., $\mathbf{r}=\left[\begin{array}{llll}r_{1} & r_{2} & \ldots & r_{N}\end{array}\right]^{T}$ and $\boldsymbol{\theta}=\left[\begin{array}{llll}\theta_{1} & \theta_{2} & \ldots & \theta_{N}\end{array}\right]^{T}$.
} 


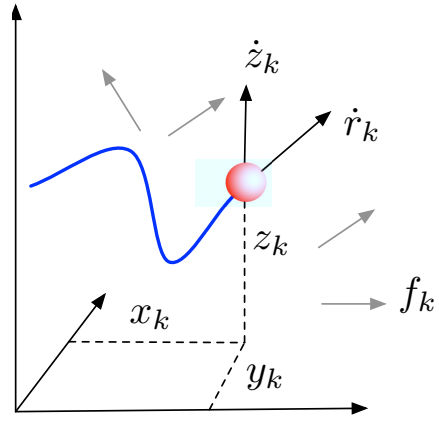

(a)

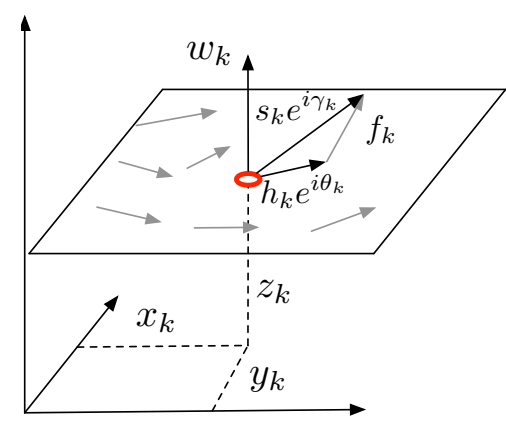

(b)

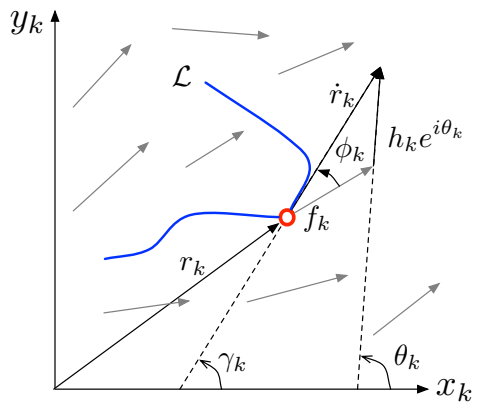

(c)

Figure 1. (a) Particle position represented in three dimensions; (b) flow-relative and total velocity orientation coordinates; and (c) total horizontal velocity of the $k^{\text {th }}$ particle.

feedback control when the states are known and observer-based feedback otherwise. The $k^{\text {th }}$ particle's climb rate control is $\dot{z}_{k}=w_{k}(\mathbf{r}, \boldsymbol{\theta}, \mathbf{z})$. The flow-free equations of motion of the $k^{\text {th }}$ particle are

$$
\begin{aligned}
& \dot{r}_{k}=h_{k} e^{i \theta_{k}} \\
& \dot{\theta}_{k}=u_{k} \\
& \dot{z}_{k}=w_{k} .
\end{aligned}
$$

The model (1) is augmented by including a time-invariant horizontal flowfield whose magnitude varies with altitude. A wind shear model such as this can adequately represent many environmental systems of interest. For example, a hurricane exhibits wind shear at low altitudes along the vertical profile of the storm as shown ${ }^{\mathrm{b}}$ in Figure 2(a). Assuming the flowfield is separable into horizontal and vertical terms, the flowfield model is

$$
f_{k}=f\left(r_{k}, z_{k}\right)=q\left(z_{k}\right) g\left(r_{k}\right) \in \mathbb{C},
$$

where $g\left(r_{k}\right) \in \mathbb{C}$ is a continuously differentiable, unit vector characterizing the horizontal flowfield direction. The function $q\left(z_{k}\right) \in \mathbb{R}^{+}$describes the dependence of the flow magnitude on altitude. The authors adopt the following model of $q\left(z_{k}\right): 4,5,19,29$

$$
q_{k}=q\left(z_{k}\right)=W_{r e f} \frac{\ln \left(z_{k} / h_{0}\right)}{\ln \left(z_{r e f} / h_{0}\right)},
$$

where $W_{\text {ref }}$ is the reference windspeed at reference altitude $z_{r e f}$ and $h_{0}$ is a shaping parameter of the vertical gradient(see Figure 2(b)). We assume the flow-relative speed of the $k^{\text {th }}$ particle is greater than the maximum flowspeed, i.e., $h_{k}>q_{k}$, so that each particle can maintain forward progress at any altitude. Note for the model (3), $\partial q_{k} / \partial z_{k}>0$.

\footnotetext{
${ }^{\mathrm{b}}$ Frankline, J. L., Black, M. L., and Valde, K, "Eyewall Wind Profiles in Hurricanes Determined By GPS Dropwindsondes", http://www.nhc.noaa.gov/aboutwindprofile.shtml, 17 July 2012.
} 


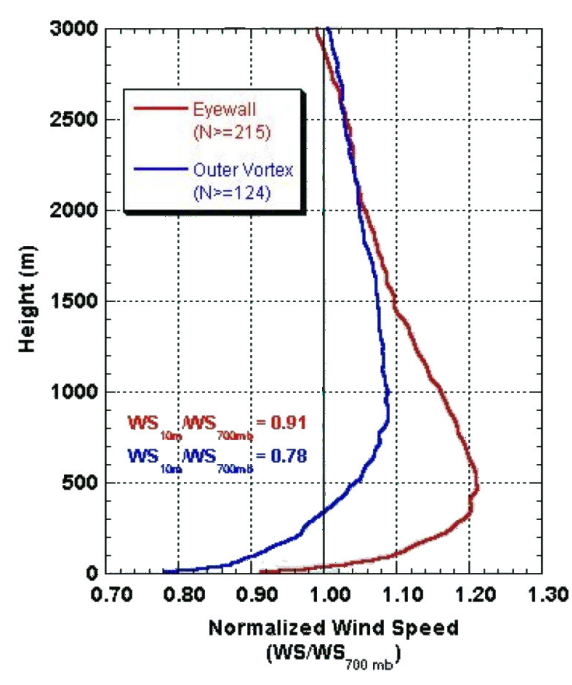

(a)

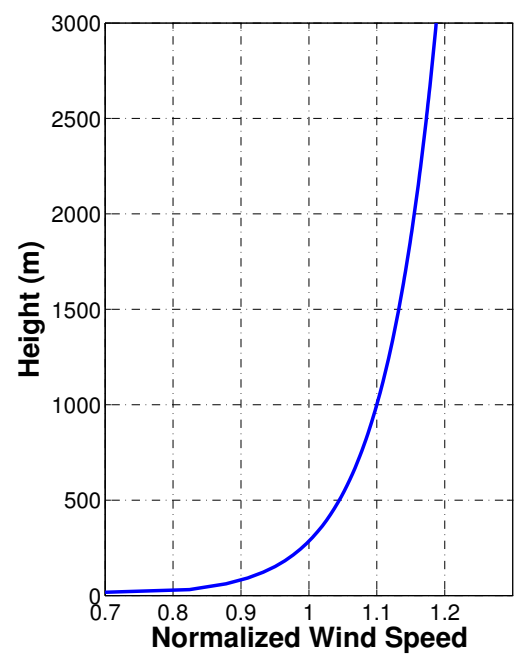

(b)

Figure 2. (a) The mean windspeed profile of a hurricane versus altitude; ${ }^{\text {b }}$ (b) wind shear model (3).

In the presence of flowfield $f_{k}$, each particle's velocity is represented by the vector sum of its velocity relative to the flow and the flow velocity relative to the ground, as shown in Figure 1(b). The equations of motion of the $k^{\text {th }}$ particle become ${ }^{6}$

$$
\begin{aligned}
& \dot{r}_{k}=h_{k} e^{i \theta_{k}}+f_{k} \\
& \dot{\theta}_{k}=u_{k} \\
& \dot{z}_{k}=w_{k},
\end{aligned}
$$

where $\dot{h}_{k}=0$. (Note that if a vertical component of the flow were included, it could be cancelled by $w_{k}$.)

For the purpose of path horizontal planning in a flowfield, one desires control of the horizontal component of the inertial velocity rather than the horizontal velocity relative to the flow. For this reason let the magnitude and direction of the total horizontal velocity be defined by, ${ }^{6}$

$$
\begin{aligned}
& s_{k} \triangleq\left|h_{k} e^{i \theta_{k}}+f_{k}\right| \\
& \gamma_{k} \triangleq \arg \left(h_{k} e^{i \theta_{k}}+f_{k}\right),
\end{aligned}
$$


such that from Fig. 1(c) we have

$$
\begin{aligned}
s_{k} \cos \gamma_{k} & =h_{k} \cos \theta_{k}+\left\langle f_{k}, 1\right\rangle \\
s_{k} \sin \gamma_{k} & =h_{k} \sin \theta_{k}+\left\langle f_{k}, i\right\rangle
\end{aligned}
$$

Taking the time derivative of (7) and (8) with $\dot{h}_{k}=0$ gives

$$
\begin{aligned}
& \dot{s}_{k} \cos \gamma_{k}-s_{k} \dot{\gamma}_{k} \sin \gamma_{k}=\dot{\theta}_{k} h_{k} \sin \theta_{k}+\left\langle\dot{f}_{k}, 1\right\rangle \\
& \dot{s}_{k} \sin \gamma_{k}-s_{k} \dot{\gamma}_{k} \cos \gamma_{k}=\dot{\theta}_{k} h_{k} \cos \theta_{k}+\left\langle\dot{f}_{k}, i\right\rangle,
\end{aligned}
$$

where $\dot{f}_{k}=\frac{\partial f_{k}}{\partial r_{k}} \dot{r}_{k}+\frac{\partial f_{k}}{\partial z_{k}} \dot{z}_{k}$. Solving for $\dot{s}_{k}$ in (9) and substituting the result into (10) to solve for $\dot{\gamma}_{k}$ gives

$$
\dot{\gamma}_{k}=\left(1-s_{k}^{-1}\left\langle f_{k}, e^{i \gamma_{k}}\right\rangle\right) u_{k}+\left\langle\frac{\partial f_{k}}{\partial r_{k}} e^{i \gamma_{k}}, i e^{i \gamma_{k}}\right\rangle+s_{k}^{-1}\left\langle\frac{\partial f_{k}}{\partial z_{k}}, i e^{i \gamma_{k}}\right\rangle w_{k} \triangleq \nu_{k},
$$

where $\nu_{k}$ is the steering control of the total horizontal velocity orientation. Similarly, solving (10) for $\dot{\gamma}_{k}$ and substituting into (9) to solve for $\dot{s}_{k}$ gives

$$
\dot{s}_{k}=\left\langle f_{k}, i e^{i \gamma_{k}}\right\rangle u_{k}+s_{k}\left\langle\frac{\partial f_{k}}{\partial r_{k}} e^{i \gamma_{k}}, e^{i \gamma_{k}}\right\rangle+\left\langle\frac{\partial f_{k}}{\partial z_{k}}, e^{i \gamma_{k}}\right\rangle w_{k} \triangleq \xi_{k}
$$

where $\xi_{k}$ is the control of the total horizontal speed.

Now solving for $w_{k}$ and $u_{k}$ with respect to $\nu_{k}$ and $\xi_{k}$ using (11) and (12) gives the altitude-speed control variable transformation

$$
w_{k}=\frac{\left\langle f_{k}, i e^{i \gamma_{k}}\right\rangle s_{k} \nu_{k}+\left(s_{k}-\left\langle f_{k}, e^{i \gamma_{k}}\right\rangle\right)\left(s_{k}\left\langle\frac{\partial f_{k}}{\partial r_{k}} e^{i \gamma_{k}}, e^{i \gamma_{k}}\right\rangle-\xi_{k}\right)-s_{k}\left\langle\frac{\partial f_{k}}{\partial r_{k}} e^{\left.i \gamma_{k}, i e^{i \gamma_{k}}\right\rangle\left\langle f_{k}, i e^{i \gamma_{k}}\right\rangle}\right.}{\left\langle\frac{\partial f_{k}}{\partial z_{k}}, f_{k}\right\rangle-s_{k}\left\langle\frac{\partial f_{k}}{\partial z_{k}}, e^{i \gamma_{k}}\right\rangle},
$$

and the steering control variable transformation

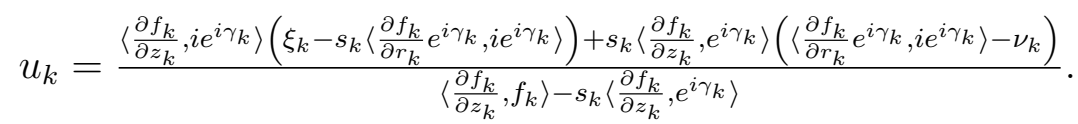

From (11) and (12) the equations of motion for a particle subject to steering and altitude control are

$$
\begin{aligned}
& \dot{r}_{k}=s_{k} e^{i \gamma_{k}} \\
& \dot{s}_{k}=\xi_{k} \\
& \dot{\gamma}_{k}=\nu_{k} \\
& \dot{z}_{k}=w_{k},
\end{aligned}
$$

\footnotetext{
${ }^{\mathrm{c}}\langle x, y\rangle \triangleq \operatorname{Re}\left(x^{*} y\right)$, where $x^{*}$ is the complex conjugate of $x$, denotes the inner product of complex numbers $x$ and $y$.
} 
where $\nu_{k}, \xi_{k}$, and $w_{k}$ are given by (11)-(13).

The mappings from $\nu_{k}$ and $\xi_{k}$ to $w_{k}$ and $u_{k}$ in equations (13) and (14) are singular when the denominator is zero. The singular conditions of (13) are derived as follows.

Lemma 1. The transformations (13) and (14) are singular in flowfield model (2) when

$$
\theta_{k}=\arg \left(f_{k}\right) \pm \pi / 2
$$

or

$$
\frac{\partial q_{k}}{\partial z_{k}}=0
$$

Proof. Equations (13) and (14) are singular when

$$
\left\langle\frac{\partial f_{k}}{\partial z_{k}}, h_{k} e^{i \theta_{k}}+f_{k}\right\rangle-\left\langle\frac{\partial f_{k}}{\partial z_{k}}, f_{k}\right\rangle=\frac{\partial q}{\partial z_{k}} h_{k}\left\langle g\left(r_{k}\right), e^{i \theta_{k}}\right\rangle=0 .
$$

Noting that $\arg \left(f_{k}\right)=\arg \left(g\left(r_{k}\right)\right)$ completes the proof.

The equations of motion in (15) can be utilized to derive decentralized multi-vehicle control algorithms. However, as shown in Lemma 1, the transformation between motion models (4) and (15) is singular for $\theta_{k}=\arg \left(f_{k}\right) \pm \pi / 2$ or when $\partial q_{k} / \partial z_{k}=0$, although the latter condition is impossible for shear model (3). To avoid this singularity, we assume bounded turn and climb rates and use a saturation function such that (4) becomes

$$
\begin{aligned}
& \dot{r}_{k}=h_{k} e^{i \theta_{k}}+f_{k} \\
& \dot{\theta}_{k}=\operatorname{sat}\left(u_{k} ; u_{\max }\right) \\
& \dot{z}_{k}=\operatorname{sat}\left(w_{k} ; w_{\max }\right),
\end{aligned}
$$

where $u_{\max }$ and $w_{\max }$ are the maximum turn and climb rates, respectively. By bounding $u_{k}$ and $w_{k}$, the $k^{\text {th }}$ particle will pass through $\theta_{k}=\arg \left(f_{k}\right) \pm \pi / 2$ with $\dot{\theta}_{k}= \pm u_{\max }$ and $\dot{z}_{k}= \pm w_{\max }$.

\section{B. Feasibility of Altitude-Induced Speed Controlled Trajectories}

While dynamic altitude control can be utilized as a means of maintaining a desired speed along a trajectory, not all speed-regulated trajectories are feasible in a spatially-varying flowfield. For example, in a uniform flowfield it is impossible to maintain a constant horizontal

speed while traveling in a circle. This section derives kinematic constraints that dictate the feasibility of a desired speed-controlled trajectory.

For a particle to travel along a given curve $\mathcal{L}$ with desired speed $s_{d}$ the flow at every point along the curve must be such that the vehicle can maintain total horizontal velocity 
$s_{d} e^{i \gamma_{k}}$ tangent to $\mathcal{L}$, as shown in Figure 1(c). The angle between $f_{k}$ given by (2) and $s_{d} e^{i \gamma_{k}}$, $\phi_{k} \triangleq \gamma_{k}-\arg \left(f_{k}\right)$, depends only on the horizontal position $r_{k}$ and not on the altitude $z_{k}$. The flow-relative horizontal velocity $e^{i \theta_{k}}$ must also satisfy the triangle equality such that $s_{d} e^{i \gamma_{k}}=e^{i \theta_{k}}+f_{k}$. From Figure 1(c) notice that the law of cosines implies

$$
h_{k}^{2}=s_{d}^{2}+\left|f_{k}\right|^{2}-2 s_{d}\left|f_{k}\right| \cos \phi_{k}
$$

which, when solved for $\left|f_{k}\right|$, gives

$$
\left|f_{k}\right|=s_{d} \cos \phi_{k} \pm \sqrt{h_{k}^{2}-s_{d}^{2} \sin ^{2} \phi_{k}}
$$

The following result is based on the argument that, if for all $r_{k} \in \mathcal{L}$, there exists a $z_{k}$ such that $\left|f_{k}\right|$ is real and positive, then $\mathcal{L}$ is feasible. Since $f_{k}$ is continuous in space, the desired $z_{k}$ is continuous along $\mathcal{L}$, assuming an arbitrarily large climb-rate saturation $w_{\text {max }}$.

Theorem 1. Trajectory $\mathcal{L}$ traversed with desired speed $s_{d}$ is feasible under the vehicle model (19) in moderate flowfield (2) if, for all $r_{k} \in \mathcal{L}$, there exists an altitude $z_{k}$ such that the quantity

$$
s_{d} \cos \phi_{k} \pm \sqrt{h_{k}^{2}-s_{d}^{2} \sin ^{2} \phi_{k}}
$$

is real, positive, and less than one, where $\phi_{k}=\gamma_{k}-\arg \left(f_{k}\right)$ and $s_{d}$ is the desired speed at $r_{k}$.

Theorem 1 is illustrated in Figure 3 for constant speed straight line and circular trajectories in the flowfield model (2). Consider a straight line trajectory in a uniform flowfield oriented toward the positive real axis, $g\left(r_{k}\right)=1$, with vertical wind shear given by (3). The trajectory is characterized by its constant velocity orientation $\gamma_{k}=\gamma_{0}$ and magnitude $s_{k}=s_{d}$. Figure 3(a) shows feasibility analysis over the space of trajectory orientations and speeds in a flowfield parameterized by $W_{\text {ref }}=0.5 \mathrm{~km} / \mathrm{min}$ and $h_{0}=4$. White areas indicate that the trajectory characterized by $\left(s_{d}, \gamma_{0}\right)$ is feasible while grey areas indicate infeasibility. The blue dot corresponds to the orientation and velocity of the trajectory in Figure 3(b).

Figures 3(c) and (d) consider a constant-speed counter-clockwise circular trajectory in a point vortex with vertical wind shear. The flowfield model is given by (2) and (3) with $g\left(r_{k}\right)=i e^{i \arg \left(r_{k}\right)}$ and shear parameters $W_{\text {ref }}=0.5 \mathrm{~km} / \mathrm{min}$ and $h_{0}=4$. Figure $3(\mathrm{c})$ illustrates a planar representation of the feasibility of circular trajectories with radius $\left|\omega_{0}\right|^{-1}=30 \mathrm{~km}$ and constant speed $s_{d}=1.5 \mathrm{~km} / \mathrm{min}$. A constant speed circular formation centered in the white area is feasible, whereas one centered in a grey area is infeasible. An example of a feasible trajectory is shown by the blue circle, centered at $c=10+0 i$. The corresponding three-dimensional trajectory that maintains $s_{d}=1.5 \mathrm{~km} / \mathrm{min}$ is shown in Figure $3(\mathrm{~d})$ and is found by solving (3) for $z_{k}$ such that $\left|f_{k}\right|$ satisfies (22). 


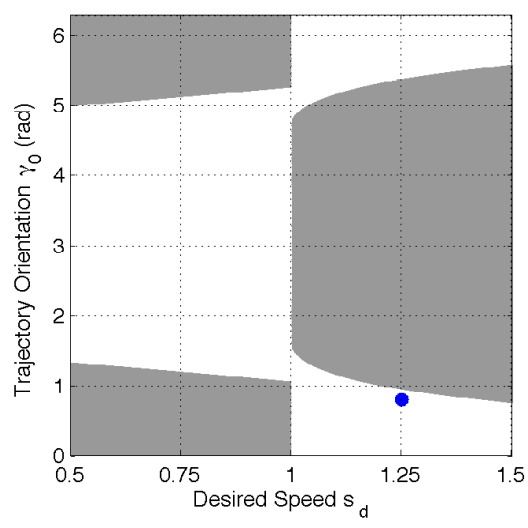

(a)

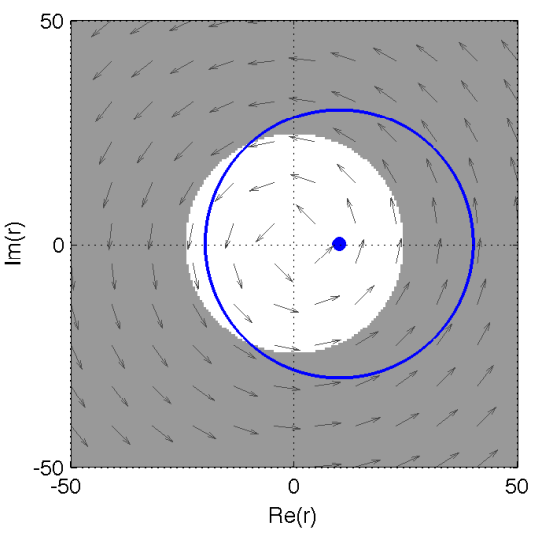

(c)

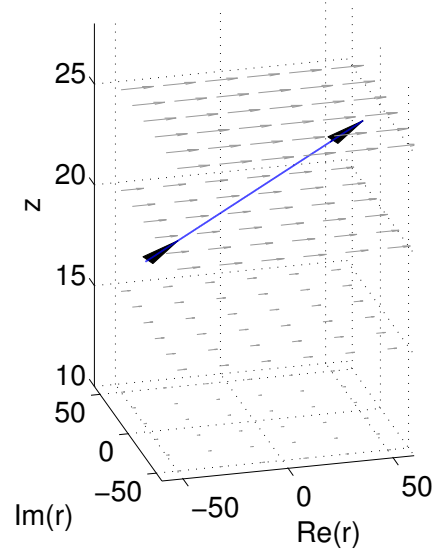

(b)

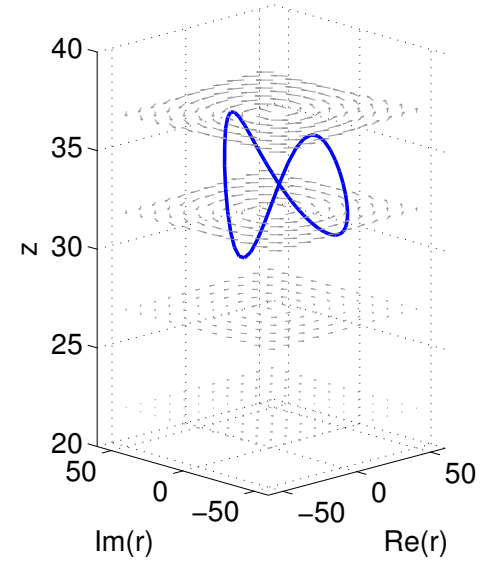

(d)

Figure 3. Feasibility regions for (a) straight line trajectories in a uniform shear flow and (c) circular trajectories in a point vortex shear flow. (b),(d) Three-dimensional trajectories corresponding to blue trajectories characterized in (a),(c).

\section{Bayesian Estimation of Shear Flow}

In order to control a vehicle's total horizontal velocity, knowledge of the flow at the vehicle's position is required. When the flow is unknown, a nonlinear estimation scheme enables each particle to estimate the flowfield at its position for use in its steering control. For the case of a flowfield of the form (2) and (3) with unknown parameters $W_{\text {ref }}$ and $h_{0}$, the authors assume the flow direction is known to simplify presentation, but direction can be estimated as well. This section presents a recursive Bayesian filter for estimating the parameters defining the wind shear model (3). The estimated shear flow is used in a dynamic altitude control described in Section IV.

Estimation of a spatiotemporal flowfield $f_{k}$ of the form (2) using noisy observations of the flow can be accomplished by assimilating the observations using a recursive Bayesian filter. 
For linear systems with Gaussian noise the optimal Bayesian filter is the Kalman filter, whereas for nonlinear systems with nonlinear noise a common Bayesian filter is a particle filter. ${ }^{20}$ In either case, the flow estimate is encapsulated in a state vector, which for example may contain the flow velocity $f\left(r_{k}(t), z_{k}(t), t\right)$ at each one of $P$ grid points. An alternative pursuit is a state vector $\Omega$ that contains only a set of $M \ll P$ parameters, from which the flowfield $f\left(r_{k}(t), z_{k}(t), t\right)$ can be reconstructed. For example, the model (3) is defined by the parameters $\boldsymbol{\Omega}=\left(W_{r e f}, h_{0}\right)$, such that the flowfield at $\vec{r}_{k / O}$ is ${ }^{\mathrm{d}} f_{k}=f\left(r_{k}, z_{k} ; \boldsymbol{\Omega}\right)$. Note that $z_{\text {ref }}$ in (3) is a reference altitude, assumed to be known. This representation provides a significant reduction in computations, making it attractive for use in a sampling scheme. Note this representation is only possible for a parameterized flowfield with a known model.

The discrete-time Bayesian formalism proceeds as follows. ${ }^{20}$ Let $\hat{\boldsymbol{\Omega}}(t)$ denote the state estimate at time $t, \tilde{\alpha}_{k}(t)$ denote the noisy observation by vehicle $k$ at time $t$, and $\mathbf{A}_{k}(t)=$ $\left\{\tilde{\alpha}_{k}(1), \ldots, \tilde{\alpha}_{k}(t)\right\}$ denote the set of observations up to time $t$. The conditional probability of the state $\hat{\boldsymbol{\Omega}}(t)$ given $\mathbf{A}_{k}(t)$ for vehicle $k$ is

$$
p\left(\hat{\mathbf{\Omega}}(t) \mid \mathbf{A}_{k}(t)\right)=\beta p\left(\tilde{\alpha}_{k}(t) \mid \boldsymbol{\Omega}(t)\right) p\left(\hat{\mathbf{\Omega}}(t) \mid \mathbf{A}_{k}(t-\Delta t)\right),
$$

where the coefficient $\beta$ can be ignored in practice as long as $p\left(\hat{\mathbf{\Omega}}(t) \mid \mathbf{A}_{k}(t)\right)$ is normalized to have unit integral over the state space. The conditional probability $p\left(\tilde{\alpha}_{k}(t) \mid \boldsymbol{\Omega}(t)\right)$ is a likelihood function that represents the probability that the state $\boldsymbol{\Omega}(t)$ generated the observation $\tilde{\alpha}_{k}(t)$. Note that $p\left(\hat{\boldsymbol{\Omega}}(1) \mid \mathbf{A}_{k}(0)\right)$ is the prior distribution, which is initialized uniformly according to known bounds.

Suppose the $k^{\text {th }}$ particle obtains the following noisy measurement of the flow magnitude at time $t$ and location $\left(r_{k}(t), z_{k}(t)\right)$ :

$$
\tilde{\alpha}_{k}(t)=q\left(z_{k}(t)\right)+\eta_{k}(t) \in \mathbb{R}
$$

where the noise $\eta_{k}(t) \sim \mathcal{N}\left(0, \sigma_{\alpha}^{2}\right)$ is normally distributed with zero mean and variance $\sigma_{\alpha}^{2}$. For each point $\Omega(t)$ in the $M$-dimensional state space, we choose the likelihood function to be a multi-variate Gaussian, i.e.,

$$
p\left(\tilde{\alpha}_{k}(t) \mid \boldsymbol{\Omega}(t)\right)=\frac{1}{2 \pi \sigma_{\alpha}^{2}} \exp \left[-\frac{1}{2 \sigma_{\alpha}^{2}}\left\|q\left(z_{k}(t) ; \boldsymbol{\Omega}(t)\right)-\tilde{\alpha}_{k}(t)\right\|^{2}\right] .
$$

Assuming measurements are taken at each time step, the conditional probability density of the state $\hat{\boldsymbol{\Omega}}(t)$ is updated with the equations of motion of the $k^{\text {th }}$ particle in an Euler

\footnotetext{
${ }^{\mathrm{d}}$ The notation $g(a, b ; \mu, \chi)$ represents a function $g(\cdot)$ that depends on the state variables $a, b$ and the parameters $\mu$ and $\chi$.
} 
integration scheme by discretizing (4) such that

$$
\begin{aligned}
r_{k}(t+\Delta t) & =r_{k}(t)+\left(e^{i \theta_{k}(t)}+f_{k}(t)\right) \Delta t \\
\theta_{k}(t+\Delta t) & =\theta_{k}(t)+u_{k}(t) \Delta t \\
z_{k}(t+\Delta t) & =z_{k}(t)+w_{k}(t) \Delta t \\
p(\hat{\boldsymbol{\Omega}}(t) \mid \boldsymbol{A}(t)) & =\beta \prod_{k=1}^{N} p\left(\tilde{\alpha}_{k}(t) \mid \boldsymbol{\Omega}(t-\Delta t)\right) p(\hat{\boldsymbol{\Omega}}(t-\Delta t) \mid \boldsymbol{A}(t-\Delta t)),
\end{aligned}
$$

where $p\left(\tilde{\alpha}_{k}(t) \mid \boldsymbol{\Omega}(t-\Delta t)\right)$ is calculated by (24), $p(\boldsymbol{\Omega}(t-\Delta t) \mid \boldsymbol{A}(t-\Delta t))$ is the prior probability density, and $\mathbf{A}(t-\Delta t)=\left\{\mathbf{A}_{1}(t-\Delta t), \ldots, \mathbf{A}_{N}(t-\Delta t)\right\}$ contains measurements from all vehicles at $t-\Delta t$. The point in parameter space corresponding to the maximum of the posterior

probability density $p(\hat{\boldsymbol{\Omega}}(t) \mid \boldsymbol{A}(t))$ gives the best estimate of the flowfield parameters $W_{\text {ref }}$ and $h_{0}$. Note that (25) assumes that the $k^{\text {th }}$ particle communicates its measurement to either a central station or to every other particle such that all vehicles have knowledge of $p(\hat{\boldsymbol{\Omega}}(t) \mid \boldsymbol{A}(t))$. Distributed versions of (25) are possible ${ }^{25}$ but beyond the scope of this paper.

\section{Dynamic Altitude Control for Motion Coordination}

This section derives a dynamic altitude control algorithm to stabilize feasible parallel and circular formations in a flowfield with wind shear. In parallel motion, the orientation of every particle's total horizontal velocity is the same. In a circular formation, every particle rotates about the same fixed point; symmetric circular formations are circular formations with equal inter-particle spacing.

\section{A. Parallel Formation Control with Speed Regulation}

This subsection extends previous work on parallel formation control algorithms ${ }^{6,8}$ by deriving a decentralized control algorithm that stabilizes the set of equal speed parallel formations in a uniform flowfield with wind shear. Particles travel with equal horizontal velocity magnitude and orientation using altitude-rate control to maintain equal horizontal speed. If the particles have the same flow-relative speed, then the steady-state altitudes are identical, otherwise they may differ.

Synchronization of the orientation of the total horizontal velocities to a desired orientation $\gamma_{0}$ corresponds to the maximum of the potential function ${ }^{6,8}$

$$
U(\gamma)=\frac{1}{2}\left|p_{\gamma}\right|^{2}+\sum_{k=1}^{N} a_{k 0} \cos \left(\gamma_{0}-\gamma_{k}\right)
$$


where

$$
p_{\gamma}=\frac{1}{N} \sum_{k=1}^{N} e^{i \gamma_{k}}
$$

and $a_{k 0}=1$ if the $k^{\text {th }}$ particle has knowledge of $\gamma_{0}$ and zero otherwise. Assuming at least one particle has knowledge of $\gamma_{0}$, the following theorem shows that choosing the steering control (11) to be the gradient of (26) establishes asymptotic stability of the parallel formation with the total velocity orientation $\gamma_{k}=\gamma_{0}$ for all $k \in\{1, \ldots, N\}$. The authors then design (12) to ensure convergence to a common reference speed $s_{k}=s_{0}$ for all $k \in\{1, \ldots, N\}$.

Theorem 2. Consider a flowfield of the form (2) that is spatially uniform with vertical shear given by (3). The model (19) with climb-rate control

$$
w_{k}=K_{s p} \operatorname{sgn}\left[\left\langle\frac{\partial f_{k}}{\partial z_{k}}, e^{i \gamma_{k}}\right\rangle-\frac{\left\langle f_{k}, i e^{i \gamma_{k}}\right\rangle\left\langle\frac{\partial f_{k}}{\partial z_{k}}, i e^{i \gamma_{k}}\right\rangle}{s_{k}-\left\langle f_{k}, e^{i \gamma_{k}}\right\rangle}\right]\left[s_{0}-s_{k}\right], \quad K_{s p}>0
$$

and turn-rate control

$$
u_{k}=\frac{-\left\langle\frac{\partial f_{k}}{\partial z_{k}}, i e^{i \gamma_{k}}\right\rangle w_{k}-K_{\gamma} s_{k}\left[\left\langle p_{\gamma}, i e^{i \gamma_{k}}\right\rangle+a_{k 0} \sin \left(\gamma_{0}-\gamma_{k}\right)\right]}{s_{k}-\left\langle f_{k}, e^{i \gamma_{k}}\right\rangle}, \quad K_{\gamma}<0,
$$

where $a_{k 0}=1$ for at least one particle, asymptotically stabilizes the set of feasible parallel formations in which $s_{k}=s_{0}$ and $\gamma_{k}=\gamma_{0}$ for all $k \in\{1, \ldots, N\}$.

Proof. Consider the time derivative of the potential function (26) along solutions of model ${ }^{6,8}$ (15)

$$
\dot{U}=\frac{\partial U}{\partial \gamma} \dot{\gamma}=\frac{1}{N} \sum_{k=1}^{N} \frac{\partial U}{\partial \gamma_{k}} \dot{\gamma}_{k}=\frac{1}{N} \sum_{k=1}^{N}\left[\left\langle p_{\gamma}, i e^{i \gamma_{k}}\right\rangle+a_{k 0} \sin \left(\gamma_{0}-\gamma_{k}\right)\right] \dot{\gamma}_{k} .
$$

Choosing the gradient control $^{8}$

$$
\dot{\gamma}_{k}=-K_{\gamma} \frac{\partial U}{\partial \gamma_{k}}=-K_{\gamma}\left[\left\langle p_{\gamma}, i e^{i \gamma_{k}}\right\rangle+a_{k 0} \sin \left(\gamma_{0}-\gamma_{k}\right)\right]
$$

and solving (11) for $u_{k}$ with $\partial f_{k} / \partial r_{k}=0$ in a uniform flowfield gives (29). Thus, (30) becomes

$$
\dot{U}=-K_{\gamma} \sum_{k=1}^{N}\left[\frac{\partial U}{\partial \gamma_{k}}\right]^{2} \geq 0, \quad K_{\gamma}<0,
$$

with equality to zero occurring at the set of critical points

$$
\Lambda=\left\{\frac{\partial U}{\partial \gamma_{k}}=0, \quad \forall k=1, \ldots, N\right\}
$$

Equation (32) ensures solutions are driven to the set $\Lambda$ of critical points of $U$ which includes 
the parallel formation $\gamma_{k}=\gamma_{0}$ (the global maximum of $U$ ). For $K_{\gamma}<0$, variation of (26) about the critical point $\gamma_{k}=\gamma_{0}$ for all $k \in\{1, \ldots, N\}$ shows asymptotic stability since $\dot{U} \geq 0$ while all other critical points are unstable.

Under control (29), the speed kinematics (12) of the $k^{\text {th }}$ particle are

$$
\dot{s}_{k}=\frac{-K_{\gamma} s_{k}\left\langle f_{k}, i e^{i \gamma_{k}}\right\rangle\left[\left\langle p_{\gamma}, i e^{i \gamma_{k}}\right\rangle+a_{k 0} \sin \left(\gamma_{0}-\gamma_{k}\right)\right]}{s_{k}-\left\langle f_{k}, e^{i \gamma_{k}}\right\rangle}+\left[\left\langle\frac{\partial f_{k}}{\partial z_{k}}, e^{i \gamma_{k}}\right\rangle-\frac{\left\langle f_{k}, i e^{i \gamma_{k}}\right\rangle\left\langle\frac{\partial f_{k}}{\partial z_{k}}, i e^{i \gamma_{k}}\right\rangle}{s_{k}-\left\langle f_{k}, e^{i \gamma_{k}}\right\rangle}\right] w_{k} .
$$

The numerator of the first term in (34) is zero in $\Lambda$ because $\left\langle p_{\gamma}, i e^{i \gamma_{k}}\right\rangle+a_{k 0} \sin \left(\gamma_{0}-\gamma_{k}\right)=0$, and with altitude control (28), equation (34) becomes

$$
\dot{s}_{k}=\underbrace{K_{s p}\left|\left\langle\frac{\partial f}{\partial z_{k}}, e^{i \gamma_{k}}\right\rangle-\frac{\left\langle f_{k}, i e^{i \gamma_{k}}\right\rangle\left\langle\frac{\partial f}{\partial z_{k}}, i e^{i \gamma_{k}}\right\rangle}{s_{k}-\left\langle f_{k}, e^{i \gamma_{k}}\right\rangle}\right|}_{\triangleq \kappa_{k} \geq 0}\left(s_{0}-s_{k}\right)
$$

The coefficient $\kappa_{k}$ is non-negative, giving the horizontal speed kinematics of the $k^{\text {th }}$ particle in $\Lambda$

$$
\dot{s}_{k}=\kappa_{k}\left(s_{0}-s_{k}\right),
$$

which are asymptotically stable about the critical point $s_{0}$ (use of the potential function $U_{s}=1 / 2 \sum_{k=1}^{N}\left(s_{0}-s_{k}\right)$ and the invariance principle [30, p.128] proves stability). Therefore, $s_{k} \rightarrow s_{0}$ for all $k \in\{1, \ldots, N\}$.

Theorem 2 stabilizes vehicle speeds to a feasible reference value $s_{0}$ assuming each vehicle has knowledge of $s_{0}$. This assumption is relaxed to the case where a subset of particles contain knowledge of $s_{0}$ by implementing a consensus algorithm on the speed control. The result is as follows.

Corollary 1. Consider a flowfield of the form (2) that is spatially uniform with vertical shear given by (3). The model (19) with climb-rate control

$$
w_{k}=K_{s p} \operatorname{sgn}\left[\left\langle\frac{\partial f_{k}}{\partial z_{k}}, e^{i \gamma_{k}}\right\rangle-\frac{\left\langle f_{k}, i e^{i \gamma_{k}}\right\rangle\left\langle\frac{\partial f_{k}}{\partial z_{k}}, i e^{i \gamma_{k}}\right\rangle}{s_{k}-\left\langle f_{k}, e^{i \gamma_{k}}\right\rangle}\right]\left[a_{k 0}\left(s_{0}-s_{k}\right)+\frac{1}{N} \sum_{j=1}^{N}\left(s_{j}-s_{k}\right)\right]
$$

where $K_{s p}>0, a_{k 0}$ is non zero for at least one particle, and turn-rate control (29), asymptotically stabilizes the set of feasible parallel formations in which $s_{k}=s_{0}$ and $\gamma_{k}=\gamma_{0}$ for all $k \in\{1, \ldots, N\}$.

Proof. Theorem 2 shows that turn-rate control (29) steers horizontal particle velocity orientations to the critical set $\Lambda$ which includes the asymptotically stable critical point $\gamma_{k}=\gamma_{0}$ 


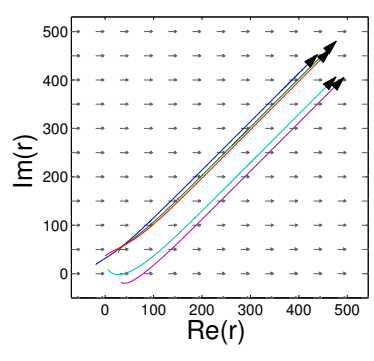

(a)

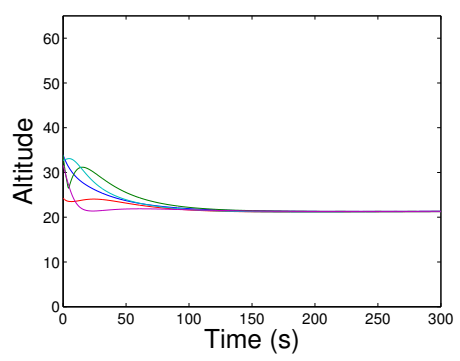

(b)

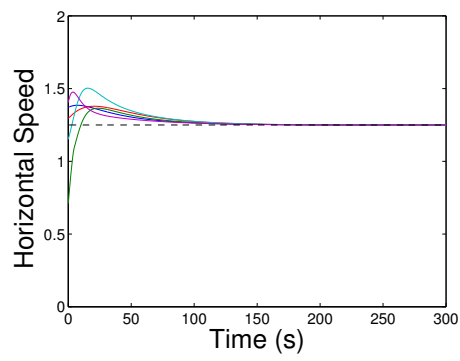

(c)

Figure 4. Illustration of particle (a) horizontal trajectories, (b) altitudes, and (c) total horizontal speeds when flow-relative particle speeds are equal.

for all $k \in\{1, \ldots, N\}$. In $\Lambda$, the speed kinematics of the $k^{\text {th }}$ particle with climb-rate control (37) are

$$
\dot{s}_{k}=\kappa_{k}\left[a_{k 0}\left(s_{0}-s_{k}\right)+\sum_{j=1}^{N}\left(s_{j}-s_{k}\right)\right],
$$

where $\kappa_{k}$ is non-negative, which represent a constant reference consensus algorithm on a directed spanning tree with reference $s_{0}$. It follows from Ren [31, Thm 3.1] that $s_{k} \rightarrow s_{0}$ for all $k \in\{1, \ldots, N\}$.

Note equations (28), (29), and (37) are well defined in a moderate flow because the denominator is never zero. ${ }^{6}$ Figure 4 shows simulation results illustrating Corollary 1 with $N=5$ particles, $K_{s p}=1$, and $K_{\gamma}=-0.1$ in a uniform flowfield with vertical shear parameterized by $W_{\text {ref }}=0.5 \mathrm{~km} / \mathrm{min}$ and $h_{0}=4$. The reference values $\gamma_{0}=\pi / 4$ radians, and $s_{0}=1.25 \mathrm{~km} / \mathrm{min}$ are chosen consistent with the feasibility analysis of Section II.B. Particles move at equal speed relative to the flow, i.e., $h_{j}=h_{k}=1 \mathrm{~km} / \mathrm{min}$ for all pairs $j, k \in\{1, \ldots, N\}$ and $a_{k 0}=1$ for two particles. Particle horizontal trajectories are shown in Figure 4(a) with the corresponding altitude and total horizontal speed shown in Figures 4(b) and 4(c), respectively. Note all particles reach consensus on not only speed but also altitude since they move at equal speed relative to the flow. Figure 5 shows simulation results illustrating Corollary 1 where flow-relative particle speeds are randomly chosen from the interval $h_{k} \in[1,1.2]$. Note that vehicles reach consensus on total horizontal speed by converging to different altitudes in order to take advantage of the wind shear.

Corollary 1 is now implemented using flowfield estimates generated by the Bayesian filter presented in Section III. The parameter estimates $\hat{\Omega}(t)=\left(\hat{W}_{\text {ref }}, \hat{h}_{0}\right)$ correspond to the maximum of the posterior probability density $p(\hat{\mathbf{\Omega}}(t) \mid \mathbf{A}(t))$. The estimated flowfield $\hat{f}_{k}$ replaces the known flowfield $f_{k}$ in the control algorithms (29) and (37). Note that the flow direction is assumed to be known, and only the parameters $\Omega=\left(W_{\text {ref }}, h_{0}\right)$ in the model (3) are estimated. The results are described as follows. 


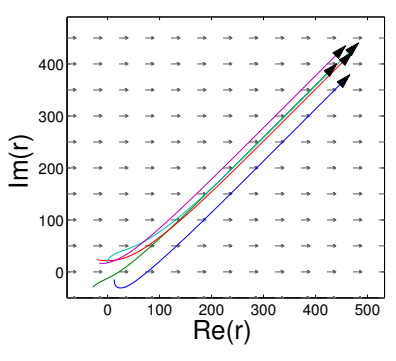

(a)

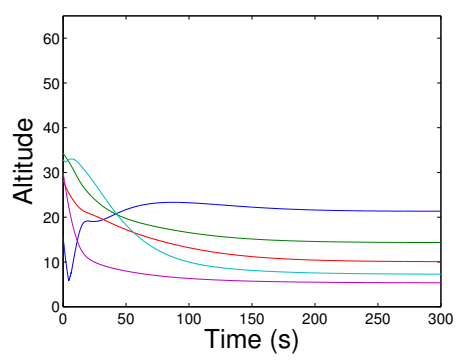

(b)

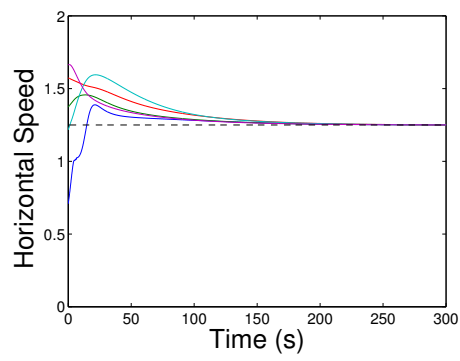

(c)

Figure 5. Illustration of particle (a) horizontal trajectories, (b) altitudes, and (c) total horizontal speeds when flow-relative speeds are in the interval $[1,1.2]$.

Proposition 1. Consider a spatially uniform flowfield with vertical shear given by (3). For the particle motion and Bayesian filtering model (25), the controls (29) and (37) calculated using flowfield estimates $\hat{f}_{k}=f\left(r_{k}, z_{k}, \hat{\mathbf{\Omega}}(t)\right)$ asymptotically stabilize the set of feasible parallel formations in which $\gamma_{k}=\gamma_{0}$ and $s_{k}=s_{0}$ for all $k \in\{1, \ldots, N\}$ and constant $s_{0}$ and $\gamma_{0}$.

Proposition 1 is justified by the decoupling of the estimation error from the control algorithm. If the recursive Bayesian filter converges around the ground truth parameters then the flowfield estimates converge to the actual flow, in which case Corollary 1 proves that vehicles asymptotically stabilize to the set of feasible parallel formations. Figure 6 shows simulation results of iterating the discrete equations of motion (25) with $N=5$ particles and flowfield estimates generated by the Bayesian filter. Figures 6(a) and 6(b) show the particle trajectories and the state-space probability density, respectively, after $t=30$ minutes. Figures 6(c) and 6(d) show the trajectories and associated probability density after $t=400$ minutes. The measurement noise is $\sigma_{\alpha}=0.3$ and the control gains are $K_{s p}=1$ and $K_{\gamma}=-0.1$. The ground truth flowfield is characterized by the parameters $W_{\text {ref }}=0.5$ and $h_{0}=4$ as shown by the white dot in Figures 6(b) and 6(d); the reference altitude is $z_{r e f}=3 \mathrm{~km}$. The maximum of the probability density is shown as a magenta dot. Note that as the vehicles continually collect measurements the probability density peaks around the true values. As this occurs the flowfield estimates become more accurate and the control algorithm steers vehicles to a parallel formation where each vehicle has the same horizontal velocity.

\section{B. Circular Formation Control with Speed Regulation}

A symmetric circular formation is a circular formation in which particles are spaced evenly about the circle. This subsection designs distributed steering and altitude controls to stabilize the set of symmetric circular formations in a point vortex with vertical shear (3).

In order to maintain uniform spacing around the horizontal projection of the formation, 


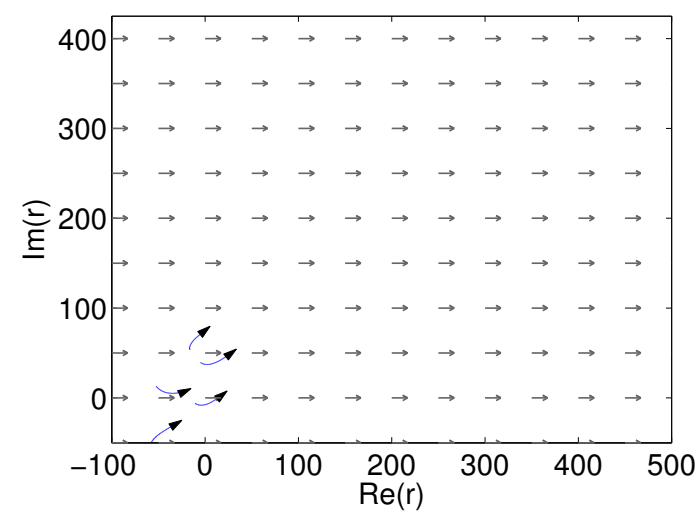

(a)

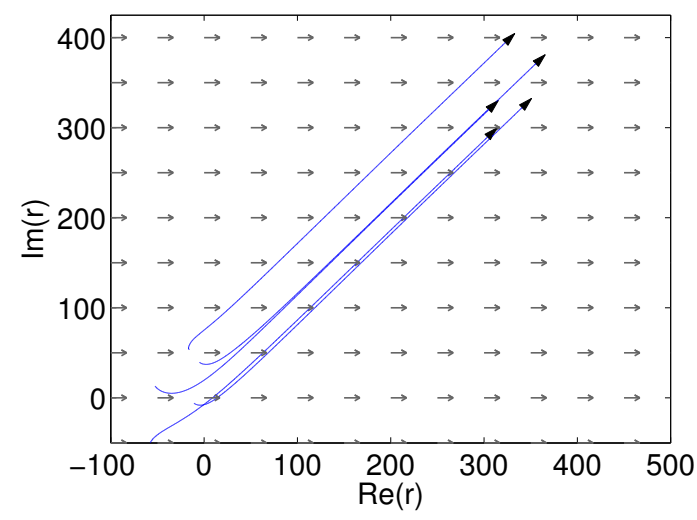

(c)

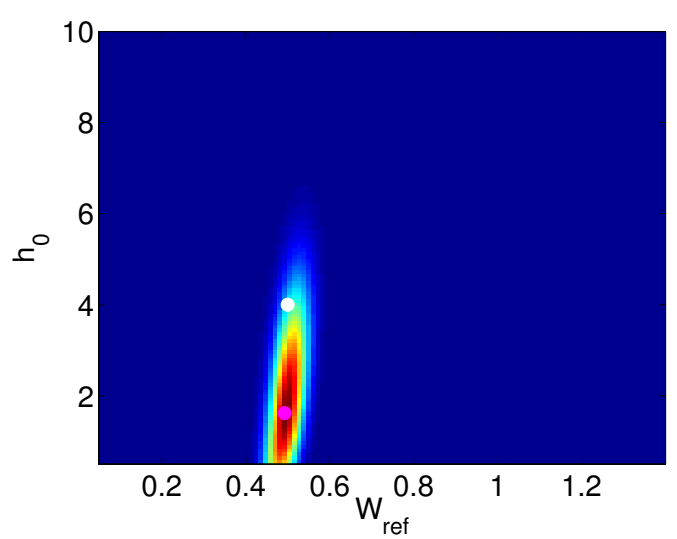

(b)

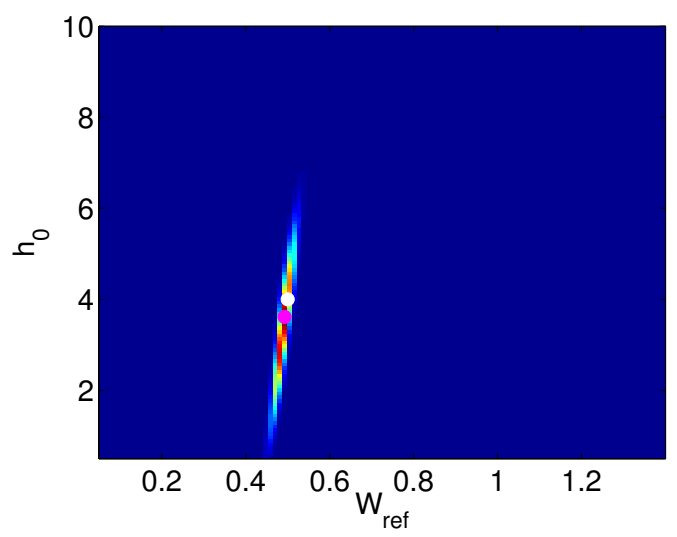

(d)

Figure 6. Simulation illustrating Proposition 1 with $N=5$ particles. Particle horizontal trajectories and state-space probability density of estimated parameters at $(\mathbf{a}, \mathbf{b}) t=30$ minutes and $(\mathbf{c}, \mathbf{d}) t=400$ minutes.

the particles regulate their total horizontal speed using altitude control. Without loss of generality, we assume the vortex is centered at the origin of the reference frame $\mathcal{I}$ such that the flowfield is

$$
f_{k}=q\left(z_{k}\right) i e^{i \arg \left(r_{k}\right)} \in \mathbb{C}
$$

with $q\left(z_{k}\right)$ given by $(3)$.

The $k^{\text {th }}$ particle's instantaneous center of rotation ${ }^{6}$ in a flowfield is

$$
c_{k} \triangleq r_{k}+i \omega_{0}^{-1} e^{i \gamma_{k}}
$$

where $\left|\omega_{0}\right|^{-1}$ and the speed $s_{k}$ determine the radius of the circle. The time derivative of (40) reveals that the control $^{8}$

$$
\nu_{k}=\omega_{0} s_{k},
$$


yields $\dot{c}_{k}=0$ and the particle is steered in a circle about a fixed center $c_{k}$. Let $\boldsymbol{c}=$ $\left[c_{1}, c_{2}, \ldots c_{n}\right]^{T}$ be the $N \times 1$ vector of circle centers. To steer particles to a common reference center $c_{0}$, we adopt the potential function ${ }^{6,8,9}$

$$
S(\boldsymbol{r}, \boldsymbol{\gamma})=\frac{1}{2}\langle\mathbf{c}, P \mathbf{c}\rangle+\frac{1}{2} \sum_{k=1}^{N} a_{k 0}\left|c_{k}-c_{0}\right|^{2}
$$

where $P=\operatorname{diag}\{\mathbf{1}\}-\frac{1}{N} \mathbf{1 1}^{T}$ corresponds to all-to-all communication ${ }^{\mathrm{e}}$ between particles and $a_{k 0}=1$ if the $k^{\text {th }}$ particle is informed of $c_{0}$ and zero otherwise. If $a_{k 0}=0$ for at most $N-1$ particles then the control ${ }^{6}$

$$
\nu_{k}=\omega_{0}\left[s_{k}+K\left(\left\langle e^{i \gamma_{k}}, P_{k} \boldsymbol{c}\right\rangle+a_{k 0}\left\langle e^{i \gamma_{k}}, c_{k}-c_{0}\right\rangle\right)\right], \quad K>0
$$

drives particles to a circular formation about $c_{0}$ with radius $\left|\omega_{0}\right|^{-1}$.

The control algorithm (43) is extended to stabilize symmetric circular formations in a non-uniform flowfield by regulating particle speed using altitude control. The phase of the $k^{\text {th }}$ particle represents its progress about the circular formation. ${ }^{8}$ In the limit that $s_{k}$ is constant, the phase of the $k^{\text {th }}$ particle satisfies

$$
\psi_{k}=\gamma_{k}
$$

in which case the period of rotation is $T=2 \pi /\left(\omega_{0} s_{k}\right)$.

Let $U(\boldsymbol{\psi})$ be a rotationally symmetric phase potential. Rotational symmetry of $U(\boldsymbol{\psi})$ implies $\sum_{k=1}^{N} \frac{\partial U}{\partial \psi_{k}}=0 .{ }^{6,8,9}$ This paper arbitrarily chooses to coordinate particle phases on an $(M, N)$-pattern potential, $U(\boldsymbol{\psi}){ }^{6,8,9}$ The $(M, N)$-pattern, where $N$ is divisible by $M$, defines an arrangement of $M$ uniformly spaced clusters of $N / M$ vehicles. The splay formation corresponds to $M=N$ in which each cluster contains one vehicle. (For an in-depth analysis of rotationally symmetric phase potentials refer to Sepulchre et al. ${ }^{8}$ )

The time derivative of the composite potential ${ }^{6}$

$$
V(\mathbf{r}, \boldsymbol{\gamma})=S(\boldsymbol{r}, \boldsymbol{\gamma})+\frac{T}{2 \pi} U(\boldsymbol{\psi})
$$

is negative semi-definite under the steering control

$$
\nu_{k}=\omega_{0} s_{k}\left[1+K_{\gamma}\left(s_{k}\left\langle e^{i \gamma_{k}}, P_{k} \mathbf{c}\right\rangle+a_{k 0}\left\langle e^{i \gamma_{k}}, c_{k}-c_{0}\right\rangle-\frac{\partial U}{\partial \psi_{k}}\right)\right]
$$

giving the following result.

\footnotetext{
${ }^{\mathrm{e}}$ Extensions to limited communication topologies are possible ${ }^{27}$ but beyond the scope of this work.
} 
Theorem 3. The particle model (19) in flowfield (2) with climb rate control $w_{k}$ given by (13) with

$$
\xi_{k}=K_{s p}\left(s_{0}-s_{k}\right), \quad K_{s p}>0,
$$

and steering control $u_{k}$ given by (14) with $\nu_{k}$ given by (46) stabilizes the set of feasible symmetric circular formations centered at $c_{0}$ with radius $\left|\omega_{0}\right|^{-1}$ in which particle separation is determined by the critical points of $U(\boldsymbol{\psi}), s_{k}=s_{0}$ for all $k \in\{1, \ldots, N\}$, and $s_{0}>0$ constant reference speed.

Proof. Consider the positive semi-definite composite potential function formed with (45) such that

$$
V_{c}(\mathbf{r}, \boldsymbol{\gamma}, \mathbf{s})=V(\mathbf{r}, \boldsymbol{\gamma})+\frac{1}{2} \sum_{k=1}^{N}\left(s_{0}-s_{k}\right)^{2} .
$$

The time derivative of (48) is

$$
\dot{V}_{c}=\dot{V}-\sum_{k=1}^{N}\left(s_{0}-s_{k}\right) \xi_{k}
$$

where $\dot{V}$ is negative semi-definite under $\operatorname{control}^{8}$ (46). With speed control (47) equation (49) becomes

$$
\dot{V}_{c}=\dot{V}-K_{s p} \sum_{k=1}^{N}\left(s_{0}-s_{k}\right)^{2} \leq 0 .
$$

By the invariance principle, particles converge to the largest invariant set $\Lambda$ for which $\dot{V}_{c}=0$. A circular formation centered at $c_{0}$ with radius $\left|\omega_{0}\right|^{-1}$ and vehicle spacing dictated by the minima of $U(\boldsymbol{\psi})$ is the only stable isolated equilibrium point in $\Lambda$ [8, Theorem 6], [6, Corollary $2]$ and all other isolated equilibria are unstable. Furthermore, the second term on the right hand side of (50) is zero only when $s_{k}=s_{0}$ for all $k \in\{1, \ldots, N\}$. Therefore, the only stable equilibrium for which $\dot{V}_{c} \equiv 0$ is the circular formation centered at $c_{0}$ with radius $\left|\omega_{0}\right|^{-1}$, vehicle spacing dictated by the minima of $U(\boldsymbol{\psi})$, and vehicle speed $s_{k}=s_{0}$ for all $k \in\{1, \ldots, N\}$.

Theorem 3 assumes all vehicles have knowledge of the constant reference speed $s_{0}$. This result is extended to the case where a subset of vehicles contain knowledge of $s_{0}$ by implementing a consensus algorithm on the speed control. The result is as follows.

Corollary 2. The particle model (19) in flowfield (2) with climb rate control $w_{k}$ given by (13) with

$$
\xi_{k}=K_{s p}\left(a_{k 0}\left(s_{0}-s_{k}\right)+\frac{1}{N} \sum_{j=1}^{N}\left(s_{j}-s_{k}\right)\right), \quad K_{s p}>0,
$$


where $a_{k 0}$ is non-zero for at least one particle, and steering control $u_{k}$ given by (14) with $\nu_{k}$ given by (46) stabilizes particle motion to the set of feasible symmetric circular formations centered at $c_{0}$ with radius $\left|\omega_{0}\right|^{-1}$ in which particle separation is determined by the critical points of $U(\boldsymbol{\psi}), s_{k}=s_{0}$ for all $k \in\{1, \ldots, N\}$, and constant reference speed $s_{0}>0$.

Proof. With control (51) the dynamics of the $k^{\text {th }}$ particles speed represent a constant reference consensus algorithm on a directed spanning tree where the reference signal is $s_{0}$. It follows from Ren [31, Thm 3.1] that $s_{k} \rightarrow s_{0}$, for all $k=1, \ldots, N$. With $s_{k}=s_{0}$, for all $k=1, \ldots, N$, the candidate potential function (45) is negative semi-definite under steering control algorithm (14) with $\nu_{k}$ given by (46). By the Invariance principle it follows from Sepulchre [8, Theorem 6] that particles are steered to a symmetric circular formation centered at $c_{0}$ with radius $\left|\omega_{0}\right|^{-1}$ and vehicle spacing dictated by the minima of $U(\boldsymbol{\psi})$.

Figure 7 illustrates simulation results of Corollary 2 with $N=6$ particles of equal flowrelative speed $h_{j}=h_{k}=1 \mathrm{~km} / \mathrm{min}$, circle radius $\left|\omega_{0}\right|^{-1}=30 \mathrm{~km}$, and gains $K_{\gamma}=0.1$ and $K_{s p}=1$. Particles are steered to a common center $c_{0}=10+0 i$ consistent with the feasibility analysis of Section II.B. $a_{k 0}=1$ for three particles and $s_{0}=1.5 \mathrm{~km} / \mathrm{min}$. The shear parameters of the flowfield are $W_{\text {ref }}=0.5 \mathrm{~km} / \mathrm{min}$ and $h_{0}=4$ with $z_{\text {ref }}=3 \mathrm{~km}$. Note in Figure 7 (a) that particles converge to a circular formation in which each particle maintains equal spacing along the circle. Light blue trajectories indicate the transient period while particles converge to the circular formation, shown in dark blue. Figure 7(b) shows the converged formation in three-dimensions. Note that particles oscillate in altitude to maintain constant speed, which is shown in Figures $7(\mathrm{c})$ and $7(\mathrm{~d})$, respectively. All particles converge to the same total horizontal speed.

Next the control algorithm of Corollary 2 is implemented using flowfield estimates from the recursive Bayesian filter. Figure 8 shows simulation results of (25) with $N=6$ particles and controls calculated using the estimated flowfield parameters. The shear parameters are $W_{\text {ref }}=0.5 \mathrm{~km} / \mathrm{min}$ and $h_{0}=4$ with measurement noise $\sigma_{\alpha}^{2}=0.3$. Figure 8(a) shows particle trajectories in three dimensions after $t=300$ minutes. Figure $8(\mathrm{~b})$ shows the state-space probability density of the parameter space at $t=300$ minutes. The white circle represents the ground truth parameters, whereas the magenta circle represents the point in parameter space with the highest probability. Figure 8(c) shows three-dimensional particle trajectories after $t=1500$ minutes. Note that particles circle about $c_{0}=10+0 i$ with speed $s_{0}=1.5$ $\mathrm{km} / \mathrm{min}$, and achieve equal inter-particle spacing. Flowfield estimates are provided by the recursive Bayesian filter, whose converged probability density is shown in Figure 8(d). 


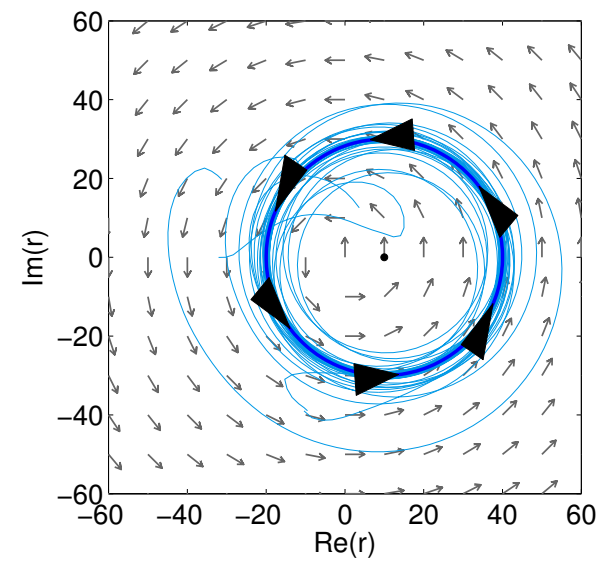

(a)

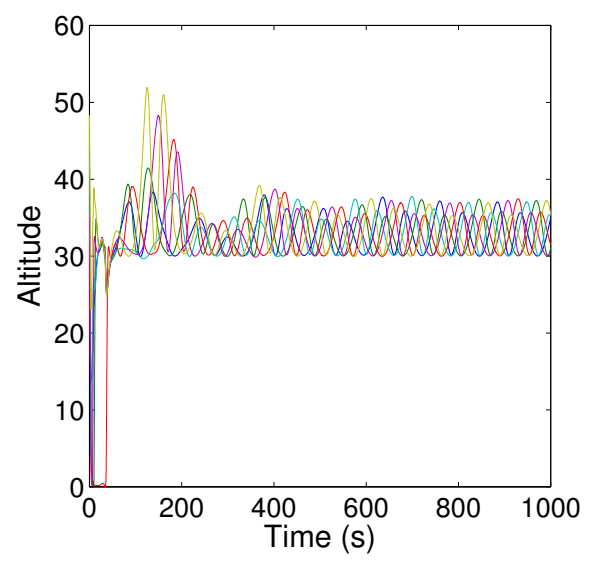

(c)

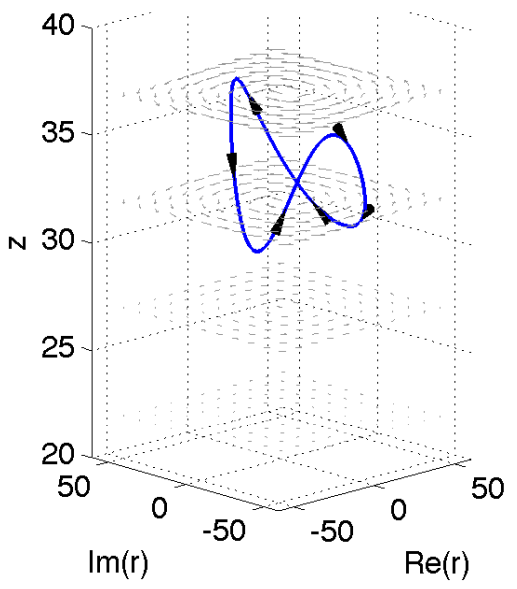

(b)

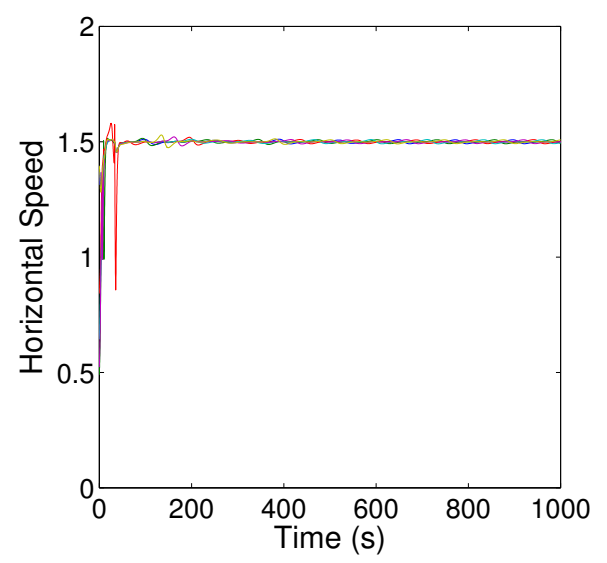

(d)

Figure 7. Simulation illustrating Corollary 2 with $N=6$ particles: (a) horizontal particle trajectories; (b) formation in three-dimensional space, (c) altitudes over time, and (d) total horizontal speeds.

\section{Conclusion}

Flowfields can be disruptive to spatiotemporal coordination in environmental sampling applications. This paper addresses how a flowfield with vertical shear can be exploited as a means of controlling vehicle speed via altitude regulation. Control of vehicle speed is used to coordinate the motion of multiple autonomous vehicles. The paper presents a threedimensional model of self-propelled particle motion in which each particle moves at constant speed relative to the flow in the horizontal plane and controls its climb rate. Decentralized multi-vehicle control algorithms are derived to stabilize feasible parallel formations with equal horizontal speed and circular formations with equal particle spacing. In addition, the authors present a recursive Bayesian filter framework to estimate parameters associated with a common wind shear model and utilize the corresponding flowfield estimate in the control 


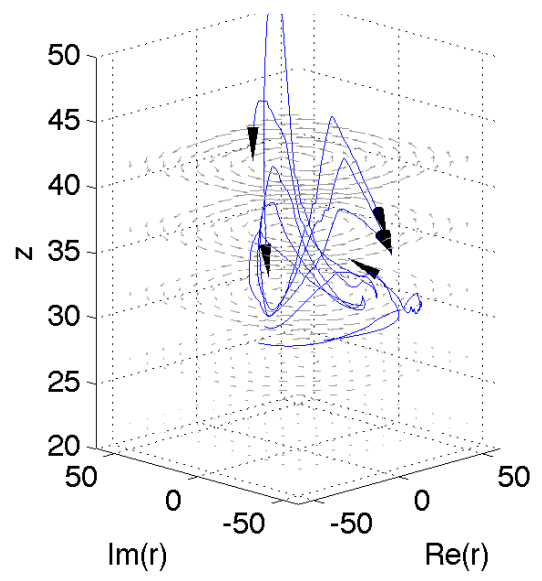

(a)

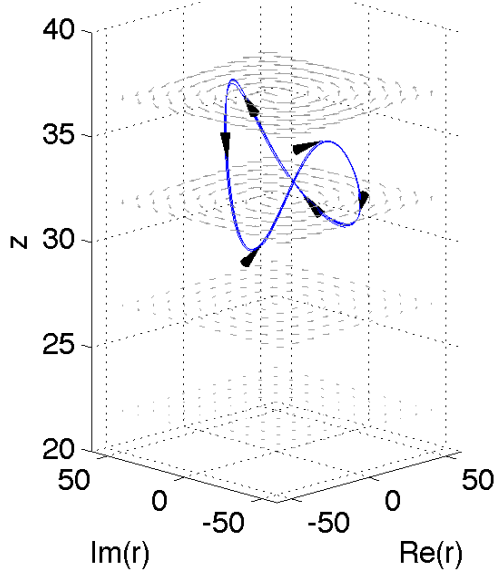

(c)

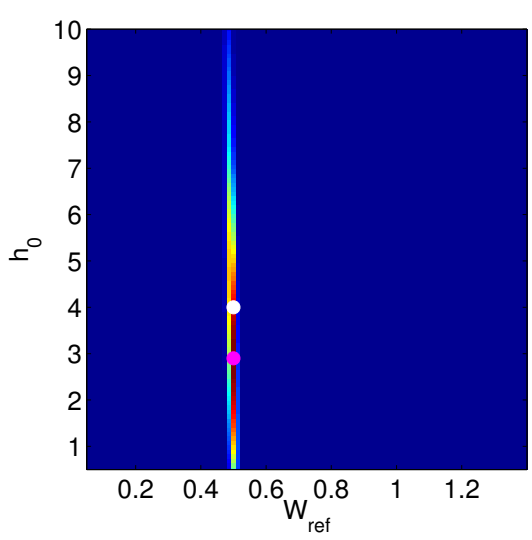

(b)

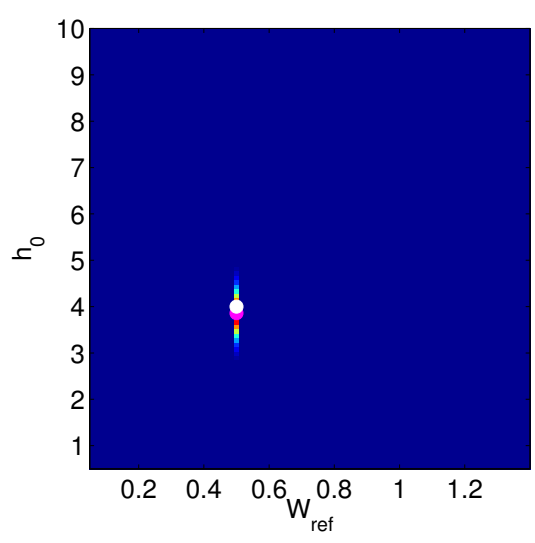

(d)

Figure 8. Simulation of symmetric circular formation control (46) with $N=6$ particles and wind shear estimation; trajectories and probability density of shear parameters at $(\mathbf{a}, \mathbf{b}) t=300$ minutes, and (c,d) $t=1500$ minutes.

algorithms. Ongoing work seeks to expand this method of control to more general flowfield and vehicle models.

\section{Acknowledgments}

The authors would like to acknowledge Steve Rasmussen, Doug Koch, and Sharan Majumdar for discussions related to this work. This material is based upon research supported by the National Science Foundation under Grant No. CMMI0928416 and the Office of Naval Research under Grant No. N00014-09-1-1058. 


\section{References}

${ }^{1}$ Lin, P., "OBSERVATIONS: The First Successful Typhoon Eyewall-Penetration Reconnaissance Flight Mission Conducted by the Unmanned Aerial Vehicle, Aerosonde," Bulletin of the American Meteorological Society, Vol. 87, 2006, pp. 1481-1483.

${ }^{2}$ Schofield, O., Kohut, J., Aragon, D., and et. al, "Slocum Gliders: Robust and Ready," Journal of Field Robotics, Vol. 24, No. 6, June 2007, pp. 473-485.

${ }^{3}$ Ákos, Z., Nagy, M., Leven, S., and Vicsek, T., "Thermal Soaring Flight of Birds and Unmanned Aerial Vehicles," Bioinspiration biomimetics, Vol. 5, 2010.

${ }^{4}$ Sachs, G., "Minimum Shear Wind Strength Required for Dynamic Soaring of Albatross," Ibis, Vol. 147, No. 1, 2005, pp. 1-10.

${ }^{5}$ Wark, K., Warner, C. F., and Davis, W. T., Air Pollution: Its Origin and Control, Addison Wesley, 3rd ed., 1998.

${ }^{6}$ Paley, D. A. and Peterson, C., "Stabilization of Collective Motion in a Time-Invariant Flowfield," AIAA Journal of Guidance, Control, and Dynamics, Vol. 32, June 2009, pp. 771-779.

${ }^{7}$ Sydney, N. and Paley, D. A., "Multi-vehicle Control and Optimization for Spatiotemporal Sampling," In Proc. of the IEEE Conference on Decision and Control, December 2011, pp. 5607-5612.

${ }^{8}$ Sepulchre, R., Paley, D. A., and Leonard, N. E., "Stabilization of Planar Collective Motion: All-to-All Communication," IEEE Transactions on Automatic Control, Vol. 52, May 2007, pp. 811-824.

${ }^{9}$ Techy, L., Paley, D. A., and Woolsey, C., "UAV Coordination on Closed Convex Paths in Wind," AIAA Journal of Guidance, Control, and Dynamics, Vol. 33, 2010, pp. 1946-1951.

${ }^{10}$ Frew, E., Lawrence, D., and Morris, S., "Coordinated Standoff Tracking of Moving Targets using Lyapunov Guidance Vector Fields," AIAA Journal of Guidance, Control, and Dynamics, Vol. 31, March 2008, pp. 290-306.

${ }^{11}$ Zhang, F., "Coordinated Patterns on Smooth Curves," Proc. of IEEE International Conference on Networking, Sensing and Control, 2006.

${ }^{12}$ Peterson, C. and Paley, D. A., "Multi-Vehicle Coordination in an Estimated Time-Varying Flowfield," AIAA Journal of Guidance, Control, and Dynamics, Vol. 34, 2011, pp. 177-191.

${ }^{13}$ DeVries, L. and Paley, D. A., "Multi-vehicle Control in a Strong Flowfield with Application to Hurricane Sampling," Journal of Guidance, Control, and Dynamics, Vol. 35, No. 3, May-June 2012, pp. 794-806.

${ }^{14}$ Bakolas, E. and Tsiotras, P., "Minimum-Time Paths for a Light Aircraft in the Presence of RegionallyVarying Strong Winds," In AIAA Infotech at Aerospace, AIAA Paper 2010-3380, Atlanta, GA, April 2010.

${ }^{15}$ Justh, E. W. and Krishnaprasad, P. S., "Natural Frames and Interacting Particles in Three Dimensions," In Proc. of the IEEE Conference on Decision and Control, December 2005, pp. 2841-2846.

${ }^{16}$ Scardovi, L., Leonard, N., and Sepulchre, R., "Stabilization of Three-Dimensional Collective Motion," Communication in Information and Systems, June 2008, pp. 15.

${ }^{17}$ Hernandez, S. and Paley, D. A., "Three-Dimensional Motion Coordination in a Time-Invariant Flowfield," In Proc. of the IEEE Conference on Decision and Control, 2009, pp. 7043-7048.

${ }^{18}$ Peterson, C. and Paley, D. A., "Distributed Estimation for Motion Coordination in an Unknown Spatiotemporal Flowfield," In Proc. of AIAA Guidance, Navigation, and Control Conference, 2011.

${ }^{19}$ Bencatel, R., Sousa, J., Faied, M., and Girard, A., "Shear Wind Estimation," In Proc. of the AIAA Guidance, Navigation, and Control Conference, Portland Oregon, August 2011. 
${ }^{20}$ Bergman, N., Recursive Bayesian Estimation Navigation and Tracking Applications, Ph.D. thesis, Department of Electrical Engineering, Linkoping University, Sweden, 1999.

${ }^{21}$ Palanthandalam-Madapusi, H. J., Girard, A., and Bernstein, D. S., "Wind-field Reconstruction Using Flight Data," In Proc. of the 2008 American Control Conference, 2008, pp. 1863-1868.

${ }^{22}$ Chao, H. and Chen, Y., "Surface Wind Profile Measurement Using Multiple Small Unmanned Aerial Vehicles," In Proc. of the 2010 American Control Conference, June-July 2010, pp. 4133-4138.

${ }^{23}$ Mulgund, S. and Stengel, R. F., "Optimal Nonlinear Estimation for Aircraft Flight Control in Wind Shear," Automatica, Vol. 32, No. 1, 1996, pp. 3-13.

${ }^{24}$ Lawrance, N. R. and Sukkarieh, S., "Autonomous Exploration of a Wind Field with a Gliding Aircraft," Journal of Guidance, Control, and Dynamics, Vol. 34, No. 3, May-June 2011, pp. 719-733.

${ }^{25}$ Lynch, K. M., Schwartz, I. B., Yang, P., and Freeman, R. A., "Decentralized Environmental Modeling by Mobile Sensor Networks," IEEE Transactions on Robotics, Vol. 24, No. 3, 2008, pp. 710-724.

${ }^{26}$ Brinon Arranz, L., Seuret, A., and Canudas De Wit, C., "Contraction Control of a Fleet Circular Formation of AUVs under Limited Communication Range," In Proc. of the 2010 American Control Conference, Baltimore, MD, June 2010, pp. 5991-5996.

${ }^{27}$ Sepulchre, R., Paley, D. A., and Leonard, N. E., "Stabilization of Planar Collective Motion with Limited Communication," IEEE Transactions on Automatic Control, Vol. 53, April 2008, pp. 706-719.

${ }^{28}$ Sepulchre, R., Paley, D. A., and Leonard, N., "Stabilization of Collective Motion of Self-Propelled Particles," In Proc. of the 16th Int. Symp. Mathematical Theory of Networks and Systems, July 2004, pp. 10.

29 "United States Military Specification MIL-F-8785C," 5 November 1980.

${ }^{30}$ Khalil, H. K., Nonlinear Systems, Prentice Hall, 3rd ed., 2002.

${ }^{31}$ Ren, W., "Multi-vehicle Consensus with a Time-varying Reference State," Systems and Control Letters, Vol. 56, 2007, pp. 474-483. 